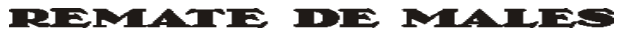

Campinas-SP, v.39, n.2, pp. 867-881, jul./dez. 2019

\title{
GÉNÉALOGIE DE L'ÉCRITURE DE JEAN BAUDRILLARD
}

\section{GENEALOGY OF JEAN BAUDRILLARD'S WRITING \\ Pierre-Ulysse Barranque ${ }^{1}$}

Résumé : Philosophe français aussi hétérodoxe que capital, Jean Baudrillard n’a pu développer son écriture qu'avec la confrontation théorique des plus grands esprits de son temps. Ainsi, aux sources de l'écriture de Baudrillard se trouve avant tout la vie d'un lecteur. Et c'est au travers de l'analyse de sa bibliothèque qu'une généalogie objective de la pensée de Baudrillard devient possible, en faisant retour sur ses analyses critiques des textes littéraires, philosophiques, anthropologiques, etc. J'essaierai de montrer dans cet article les premiers éléments d'un travail d'archives inédit, qui dévoile la pratique d'un philosophe dans son atelier textuel.

Mots-clés : Baudrillard ; philosophie française contemporaine ; archives.

Abstract : French philosopher as heterodox as capital, Jean Baudrillard could develop his writing with the theoretical confrontation of the greatest minds of his time. Thus, to the sources of Baudrillard's writing lies above all the life of a reader. And it is through the analysis of his library that an objective genealogy of Baudrillard's thought becomes possible, by reviewing his critical analyzes of literary, philosophical, anthropological texts, and so on. I will try to show in this article the first elements of an original archival work, which reveals the practice of a philosopher in his textual workshop.

Keywords : Baudrillard ; Contemporary French Philosophy ; Archives.

Pour commencer, deux mots sur l'origine du projet que j'ai consacré à la bibliothèque de Jean Baudrillard. Cela nous renvoie à l'événement que nous avons organisé avec Marine Baudrillard, et toute une équipe, en 2017 : la « Baudrillard Street One », journée consacrée à l'œuvre de

1 Doctorant en Esthétique au laboratoire EsPas (ACTE), à l'Université Paris-1 Panthéon/ Sorbonne, France: <p-u.barranque@protonmail.com>. 
Jean Baudrillard, disparu dix ans plus tôt. Marine Baudrillard m'avait alors montré le «Veblen » de Jean Baudrillard - le « Veblen », c'est-à-dire le recueil en anglais des principaux textes du sociologue américain Thorstein Veblen, sociologue qui fut l'un des précurseurs dans l'analyse des sociétés capitalistes modernes, et qui eut une grande influence sur les premiers écrits de Baudrillard (1986) : je pense notamment à son deuxième ouvrage La Société de consommation. Quelques pages de ce « Veblen » en anglais, largement annotées en français par Baudrillard, étaient d'ailleurs déjà reproduites dans l'excellent livre dirigé par Valérie Guillaume (2013) : Jean Baudrillard et le Centre Pompidou, une biographie intellectuelle. Intrigué par les milliers d'ouvrages accumulés chez Marine et Jean Baudrillard dans cette bibliothèque, une brève recherche me fit découvrir d'autres livres annotés. Et c'est ainsi que commença ce travail d'archives. Après consultation de l'ensemble des ouvrages de la bibliothèque, j'ai découvert 336 livres ou revues, sous-lignées et/ou annotées par Jean Baudrillard, et qui couvrent l'ensemble de son œuvre, puisque certains de ces ouvrages font partie des éditions commentées dans sa thèse, qui est en même temps son premier essai, à savoir Le Système des objets (BAUDRILLARD, 1978), et que d'autres figurent parmi les livres lus dans les dernières années de sa vie. Du mode d'existence des objets techniques, de Gilbert Simondon (1958), cité dans l'introduction du Système des objets en 1968, jusqu'à La haine de la démocratie de Jacques Rancière (2005), on a là une photographie d'une pensée qui apparaît dans les années 1960, et qui suit, décennies après décennies, les premières années du XXIe siècle.

J'ai également retrouvé l'équivalent de quatre cartons d'archives. Le contenu de ces archives est très important, puisque le premier carton est composé d'écrits de pataphysiciens collectionnés par Jean Baudrillard au fil des années, et les trois autres contiennent une vaste documentation, des articles de journaux, des articles de revues, des entretiens de Jean Baudrillard, des lettres d'universitaires, mais aussi les ébauches de différents manuscrits sur lesquels Baudrillard a travaillé dans les années 1990-2000. Un de ces cartons a pour objet la publication du Complot de l'art (BAUDRILLARD, 2005), et les débats qui ont suivi à la suite de ce texte, avec de nombreux articles, des correspondances, différents types de réactions d'intellectuels et de lecteurs concernant cette polémique. Deux autres cartons concernent les attentats du 11 Septembre, même si on y trouve également les premières versions de certains articles tardifs pour Libération, et plusieurs manuscrits publiés par les éditions Sens \& 
Tonka. Autrement dit, il s'agit ici des sources, des travaux préparatoires et des manuscrits des deux livres de Baudrillard sur l'attentat des Twin Towers : Power Inferno (BAUDRILLARD, 2002) et L'esprit du terrorisme (BAUDRILLARD, 2002).

La grande diversité des documents dans son analyse du phénomène terroriste manifeste l'objectif explicite de ce travail : articles de référence des grands organes de presse nationaux et internationaux, coupure de photos issues de Paris Match, articles sur les philosophes Agamben, Sloterdijk ou Zizek analysant l'événement, ces sources nous permettent de comprendre comment Baudrillard construisait sa pensée à la fin de sa vie : d'une part en se confrontant avec les analyses conceptuelles des auteurs contemporains les plus importants, et d'autre part en étudiant les images et les textes des médias de masse, afin de saisir ce qui est effectivement montré dans cette communication mass-médiatique. On y perçoit un Baudrillard essayant de penser ce que personne ne pense, ce qui échappe à ses contemporains, tout en s'efforçant de comprendre ce que tout le monde voit. Ces archives témoignent d'une méthode de travail capable d'éclairer les enjeux de l'époque, et dont pourrait s'inspirer de nombreux intellectuels contemporains : ne pas transiger sur l'exigence radicale de la théorie, tout en faisant de la philosophie une arme pour penser l'actualité historique la plus immanente.

Avec cette bibliothèque, nous apercevons une photographie tant des lectures les plus importantes de Jean Baudrillard que de sa méthode de travail. Quand je dis que les archives de la bibliothèque sont une photographie, j'entends par-là que nous ne pouvons bien sûr pas connaître l'ensemble des ouvrages lus par Baudrillard, ni même présumer de l'importance pour lui de textes qui n'auraient pas été cités dans ses livres, annotés dans la bibliothèque, ou qui auraient disparues, qui aurait été offerts, etc. Nous ne pouvons connaître que les livres annotés dont l'importance était telle qu'il les a gardés auprès de lui. J'ai donc réalisé un inventaire qui doit nécessairement aux aléas d'une vie passée à créer une pensée aux quatre coins du monde. Mais cet inventaire manifeste aussi l'état de la philosophie de Baudrillard au moment où celui-ci a disparu. Comme une photographie, cette bibliothèque est la vérité accidentelle d'un instant, ce qui nous dit déjà beaucoup de son œuvre.

J'ai ainsi sélectionné tous les livres annotés ou sous-lignés par Jean Baudrillard. La plupart des livres sont seulement sous-lignés, mais les livres annotés, qui font donc l'objet d'un commentaire spécifique, sont 
bien souvent des textes cruciaux pour le philosophe : soit qu'il s'agisse d'ouvrages dont la lecture est déterminante dans la construction de la pensée de Baudrillard, des ouvrages écrits par les auteurs qui ont des problématiques communes avec ce dernier : je pense entre autres à Elias Canetti, à Herbert Marcuse, à Henri Lefebvre, à Georges Bataille ; ou bien qu'il s'agisse d'auteurs contemporains de Jean Baudrillard avec lesquels le philosophe partage de vastes polémiques intellectuelles : c'est le cas notamment de Gilles Deleuze et Félix Guattari, de Louis Althusser, de Maurice Godelier et de Michel Foucault.

Il m’a semblé tout aussi important de sélectionner les passages simplement sous-lignés par Jean Baudrillard queceux qui sont commentés, car les passages sous-lignés sont l'expression d'un choix du philosophe dans sa lecture, et participent donc d'une véritable analyse. Même si les commentaires annotés de Baudrillard visent souvent très justes, et ne sont nullement de l'ordre de la paraphrase, un développement conceptuel repéré et sous-ligné dans un essai peut être tout aussi explicite. Je ne crois pas qu'il faille être ici fétichiste des mots, mais que l'on peut découvrir la lecture attentive de Baudrillard rien qu'en suivant ce qui a été sous-ligné par celui-ci dans un ouvrage. Il en est ainsi par exemple de L'Ethique d'Alain Badiou (1996), très précisément sous-ligné tout au long du livre, dans une lecture qui identifie les articulations conceptuelles de l'essai. Ce qui nous permet de dire que si Alain Badiou déclare ne pas avoir étudié la pensée de Jean Baudrillard (PENOT-LACASSAGNE, 2015, pp. 135-136), l'inverse n'est pas vrai. Baudrillard est toujours allé au-devant des penseurs de son époque, y compris de ceux qui semblaient les plus éloignés de sa philosophie, dans une curiosité intellectuelle sans limite.

Certaines notes, aussi laconique soient-elles, sont très significatives et en disent long sur l'interprétation générale d'un auteur. Par exemple, lisant Le théâtre et son double d'Antonin Artaud (1964), Baudrillard donne de nouveaux titres à certains textes de ce recueil. Ainsi, le texte « le théâtre alchimique » est rebaptisé par Baudrillard : «alchimiothérapie ». De même, «Sur le théâtre balinais » devient « Sur le rituel ». La question du théâtre en tant que tel s'efface dans la lecture que Baudrillard fait d'Artaud, et avec cette nouvelle dénomination le philosophe assimile l'essai fondateur du " théâtre de la cruauté » à une authentique théorie critique, plutôt qu'à un nouveau type de dramaturgie. En même temps qu'il réintitule les articles d'Artaud, Baudrillard semble peu convaincu qu'il faille circonscrire ces réflexions à la seule pratique du théâtre. C'est 
pourquoi il renvoie en note le dramaturge aux théories postérieures de Bataille, et l'associe par cet acte à une constellation d'auteurs qui seront au fondement de sa pensée.

En effet, à la fin de l'ouvrage de l'essayiste américaine Susan Sontag (1979), La photographie, Baudrillard ajoute à la main une série de noms d'auteurs à la suite des titres d'ouvrages déjà publiés par l'éditeur de Sontag : Première colonne : «Baudelaire, Benjamin, Barthes, Bataille, Borges. » Que des auteurs qui commencent par la lettre de B, comme le remarquait déjà Baudrillard lui-même (BAUDRILLARD, 2001, p. 17).

Deuxième colonne : « Baudelaire, Artaud, Rimbaud ». « Baudelaire » est cité à nouveau, mais on s'étonne presque de l'absence de Jarry. C'est que ces trois auteurs ne sont pas choisis au hasard, dans la mesure où ils forment une anagramme phonétique. Le « Baud- » de Baudelaire, plus le « Ri-» de Rimbaud, et le « Ar-» d'Artaud composent le nom de Baudrillard, qui voyait dans ces trois écrivains les trois références onomastiques qui structurent sa pensée. ${ }^{2}$ Cette anagramme sur les patronymes nous rappelle qu'un écrivain véritable est toujours dans une réinvention de son nom. Pour pouvoir écrire, il faut pouvoir s'écrire soi-même d'une façon nouvelle. Choisir le sens de son nom, c'est créer une nouvelle logique du signifiant, donc une nouvelle généalogie pour le sujet de l'écriture. Baudrillard, à la différence de nombreux auteurs, ${ }^{3}$ était conscient de ce fait, et a ainsi transformé le nom familial hérité en pseudonyme littéraire. C'est la condition de possibilité de l'écriture d'un « je » qui est alors réellement devenu « un autre».

Troisième et dernière colonne, on va vers l'essentiel : " Nietzsche, Hölderlin ». Ces trois colonnes des auteurs de référence, qui sont ceux qu'évoque Baudrillard au début du livre d'entretien avec François L'Yvonnet D'un fragment l'autre, nous donne un indice sur la pensée du philosophe. Il s'agit d'une pensée qui englobe la grande philosophie, les apports des sciences humaines et la littérature. On y trouve bien sûr : Nietzsche, Benjamin, Barthes, Bataille, Borges, Artaud. Mais il ne faut jamais oublier qu'en-deçà, ou au-delà, de ces penseurs, de ces romanciers, de ces théoriciens, il y a la puissance radicale de la poésie, de la poésie de

2 Cette anecdote m’a depuis été confirmée par Marc Guillaume, grand ami, compagnon intellectuel, et éditeur de Jean Baudrillard, avec lequel il a coécrit : Figures de l'altérité (BAUDRILLARD ; GUILLAUME, 1998).

3 Sur cette question, je renvoie à la poétique onomastique de Serge Pey (2003), développée à propos de nombreux écrivains, et notamment Octavio Paz. 
Baudelaire, de Rimbaud, et surtout de Hölderlin. Les textes de Baudrillard visent, dans la pensée, à avoir la même puissance de métamorphose que les textes poétiques. C'est un idéal d'écriture vers lequel il tendra de plus en plus d'années en années, et qui est particulièrement visible dans les Cool Memories, comme dans ses derniers essais.

La bibliothèque de Baudrillard est bien entendu plurilingue. La très grande majorité des livres sont en français, mais certains ouvrages très importants pour le philosophe sont en anglais ou en allemand, et même en italien. Nous nous souvenons que Jean Baudrillard était germaniste de formation, qu'il fut le traducteur des Dialogues d'exilés de Bertolt Brecht (1965), qu'il est le co-traducteur de L'Idéologie allemande de Marx et Engels (1968) aux Editions Sociales, qu'il a traduit plusieurs ouvrages de Peter Weiss, ainsi que des poèmes de Hölderlin, tels que nous pouvons les retrouver dans le Cahier de l'Herne (L'YVONNET, 2004, pp. 293-315) consacré à Jean Baudrillard.

Le « don des langues » de Jean Baudrillard participe de l'originalité de sa pensée, et le distingue aussi dans la constellation des philosophes français de son époque. Tout d'abord, son plurilinguisme lui permet de lire dans la langue originale des auteurs qui sont souvent méconnues alors en France. Par exemple, Baudrillard lit et cite très tôt Walter Benjamin (1966), bien avant que cet auteur soit reconnu à sa juste valeur dans l'université française. Il annoteainsi le deuxième tomedes Euvres choisies en allemand du philosophe berlinois. Et nous avons vu l'attachement théorique jamais démenti de Baudrillard pour Benjamin. De même, Baudrillard lit en anglais l'essai révolutionnaire de l'anthropologue américain Marshall Sahlins (1972) Stone age economics, traduit en français par Age de pierre, âge d'abondance, L'économie des sociétés primitives, ouvrage qui modifie radicalement notre conception des rapports économiques dans les sociétés de chasseurs-cueilleurs, et conteste la téléologie du marxisme dominant à cette époque.

Cette quête des textes dans la langue originale n'est pas seulement chez Baudrillard une pratique de jeunesse, puisqu'il lira également Peter Sloterdijk (1995) directement en allemand dans les années 1990 : Im selben Boot, Versuch über die Hyperpolitik, en français : Dans le même bateau, Essai sur l'hyperpolitique. Il lira également peu après en anglais certains premiers essais de Slavoj Zizek (2002), auteurs à l'époque méconnu en France, et ce, bien avant qu'ils ne soient traduits en langue française : notamment Welcome to the Desert of the Real [Bienvenue dans le désert 
$d u$ réel], qui est un essai important pour Baudrillard dans la mesure où Zizek y développpe une tentative d'analyse philosophique des attentats du 11 Septembre. ${ }^{4}$ Cet essai fait partie des lectures préparatoires de Baudrillard à sa propre réflexion sur les attentats, et c'est aussi en regard avec les limites qu'il décèle dans la conceptualisation très hégélienne et très lacanienne de Zizek que Baudrillard développera sa réflexion sur cet événement capital dans Power Inferno et L'esprit du terrorisme. Le caractère imprévisible et inédit de cet événement suppose une pensée de l'altérité radicale que la dialectique du maître et de l'esclave de Hegel, ou la théorie du « grand Autre » de Lacan, ne saurait résoudre. D’où la nécessité de développer une conceptualisation nouvelle pour Baudrillard (1999), et d’approfondir ses réflexions sur une société mondiale où « l'échange » est devenu « impossible».

On remarquera également sa lecture dans les deux langues, et sous différentes traductions, des Aphorismes de Georg Christoph Lichtenberg, révélatrice de la passion de Baudrillard pour la forme fragmentaire de l'écriture, qu'il développera pendant plus de 20 ans dans les différents volumes des Cool Memories. Il y a ses lectures de Marx, Nietzsche et Freud directement en allemand. Lisant la grande pensée critique de langue allemande dans sa version originale, la position singulière de Baudrillard dans le courant de la philosophie française des années 19601970 nous apparaît. Au moment même où Deleuze et Foucault devienne de nouveaux passeurs de la philosophie de Nietzsche, où Lacan amorce un « retour à Freud », et qu'Althusser et les althussériens en appellent à un « retour à Marx », Baudrillard avait déjà lu ou était en train de lire tous ces différents auteurs germanophones dans leur propre langue, avec l'appui de sa formation de germaniste et de son activité de traducteur. Ce qui explique aussi sa distance vis-à-vis de ces projets philosophiques, et l'originalité des enjeux théoriques baudrillardiens dans le contexte de la philosophie française de cette époque. Dans le cas de Nietzsche et de Freud, Baudrillard prend des notes parfois dans l'original allemand et dans la traduction en français, notes qui ne se recoupent pas tout à fait et manifeste des approches différentes dans la lecture, voire très probablement dans le cas de Nietzsche des lectures à des époques très différentes. En lisant directement en allemand plutôt que de s'en remettre aux traducteurs, surtout vis-à-vis d'auteurs dont les interprétations

4 Le « désert du réel » est d'ailleurs un concept célèbre de Baudrillard (1981, p. 10), repris dans le film Matrix (1999), et tiré du début de Simulacres et simulation. 
font l'objet de véritables luttes idéologiques, Baudrillard se distingue par sa lecture à contre-courant. Dans sa lecture de Marx, et à l'opposé de nombreux philosophes marxistes dont l'interprétation semblait faire autorité à cette époque, Baudrillard semble s'attacher à comprendre la spécificité de l'économie capitaliste moderne, en opposition avec les formes plus anciennes ou non-occidentales d'économie. Ainsi, à la différence d'Althusser, c'est un Marx ethnologue que Baudrillard recherche dans sa lecture en allemand des Grundrisse (MARX, 1953), annotant les passages sur l'économie dans l'Antiquité romaine, dans l'Antiquité germanique ou dans les pays d'Orient ; comme il lira avec attention, cette fois-ci en français, « le Chapitre VI » du Capital, le « Chapitre inédit », où Marx (1971) précise de la façon la plus claire la différence entre la société moderne capitaliste et les formes économiques médiévales. Cette lecture deviendra explicite dans Le Miroir de la production, essai de Baudrillard (1973) où celui-ci confronte marxisme et ethnologie.

La lecture à la fois très précise d'Althusser (1965) par Baudrillard, et pourtant très critique, peut nous donner un exemple de la façon dont Baudrillard lisait les penseurs de son époque. En effet, Althusser envoya en 1965 à Baudrillard la première édition dédicacée des deux volumes de Lire le Capital, («à J.Baudrillard, fraternellement ») ouvrage collectif et véritable somme philosophique, où sont publiés les textes les plus importants du philosophe marxiste et de ses disciples : Etienne Balibar, Pierre Macherey, Jacques Rancière et Roger Establet. Baudrillard y manifeste déjà son analyse de "l'objet » comme « fonction-signe », tel qu'il le développera dans Le Système des objets. Là où Althusser et les althussériens perçoivent des formes économiques capitalistes, Baudrillard recherche un système sémiotique d'objets de consommation, avec une cohérence structurelle équivalente à la structure d'une langue. Après l'invention de l'Anthropologie structurale par Claude Lévi-Strauss (1958), et après l'application de cette méthodologie ethnologique à la France de l'après-guerre par Roland Barthes (1957) dans les Mythologies, ${ }^{5}$ Baudrillard prolonge cette pensée structuraliste en comprenant que par-delà la logique, en apparence exclusivement économique, de la production des biens de consommation gît une véritable organisation sémiotique. Certes le capitalisme est une économie, mais il est aussi un langage, un code, dont les objets produits sont les unités signifiantes, ce que ne semblent

5 On se rappelle du sous-titre de La société de consommation : Ses mythes, ses structures, références explicites à Lévi-Strauss et Barthes. 
pas saisir alors Althusser et les althussériens. Il est très intéressant de voir les notes de Baudrillard sur Lire le Capital : il synthétise dans le premier volume l'essentiel de la critique althussérienne de l'empirisme en science au travers de quelques commentaires, pour ensuite retourner dans le deuxième volume cet argument contre Althusser et les althussériens eux-mêmes, leur reprochant de tomber dans le piège de l'empirisme qu'il venait de dénoncer, quand il essaient de justifier l'existence d'un bon et d'un mauvais Marx : un bon Marx scientifique authentique dans Le Capital, et un mauvais Marx encore philosophe hégélien dans ses écrits de jeunesse. « Il y a là un point de vue empiriste sur la totalité conceptuelle Marx », note Baudrillard au sujet d'Althusser, reprochant même à ce dernier d'avoir un rapport à Marx semblable à une « Pythonisse » : tel la Pythie de Delphes, la grande prêtresse du culte d'Apollon, les althussériens feraient du texte marxien un discours dont on peut dire tout et son contraire, puisqu'il est toujours à interpréter. La rigueur du germaniste Baudrillard ne pouvait tout simplement pas accepter une telle méthode.

Il en va de même avec la lecture que Jean Baudrillard fait de L'AntiEdipe de Deleuze et Guattari (1972). Ce livre semble décevoir Baudrillard, non pas en soi parce qu'il critique la psychanalyse, mais parce qu'il n'est pas allé assez loin dans cette critique. Certes, L’Anti-CEdipe, ce n'est plus de la psychanalyse, mais « c'est de l'électro-analyse », écrit en note Baudrillard ; comme si, en passant d'une théorie de la pulsion libidinale chez Freud, à une théorie des « flux » et des « machines » chez Deleuze et Guattari, on n'avait pas suffisamment changé de modèle. « Flux » ou pulsions, on manque l'une des dimensions essentielles des rapports humains, qui est celle de « l'échange », et notamment de « l'échange symbolique » qui sera l'un des concepts-clés de Baudrillard : nous pensons bien sûr à L'Echange symbolique et la mort, publié en 1976. Là encore, en privilégiant le paradigme marxiste de la « production » à la question de "l'échange », tel que l'ethnologie l'a étudié, Deleuze et Guattari semblent méconnaitre selon Baudrillard la diversité des formes économiques que les peuples ont connu, dans le temps et dans l'espace. Alors que Baudrillard recherchait en lisant Marx en allemand un "Marx ethnologue », les conceptions philosophiques développées dans L'Anti-CEdipe semblent, selon lui, encore beaucoup trop focalisée sur les formes de société modernes et occidentales. C'est leur manque de connaissance précise des formes de sociétés extra-européennes qui pose le plus problème à Baudrillard, dans cet ouvrage. Là encore, Baudrillard identifie les concepts fondamentaux 
des deux auteurs : «production », «flux », « machines », et les retournent contre ces derniers. Avec le paradigme de «production » et de «flux », Deleuze et Guattari ont essayé d'être encore plus matérialistes que les marxistes, mais Baudrillard doute que cette réduction de l'activité à la «production » soit déterminante en dehors des sociétés industrielles, dès lors que l'on quitte la modernité capitaliste occidentale. Et l'Occident n'est-il pas déjà en 1972 qu'une petite partie de l'Humanité ?

Cette critique de l'eurocentrisme des intellectuels français atteindra un point culminant dans sa lecture de l'ethnologue althussérien Maurice Godelier (1971). Dans l'article signé par celui-ci au sein de l'ouvrage collectif L'anthropologie : science des sociétés primitives ?, Baudrillard voit un " remixage ethnocentrique » dans la façon qu'a alors Godelier d'appliquer les concepts du marxisme althussérien à des peuples dont les rapports sociaux sont structurés selon des critères complètement différents. C'est que dès ses premières lectures de Marx (1962), en témoigne son ouvrage annoté des Manuscrits de 1844, Baudrillard avait déjà essayé de penser ensemble les apports de la critique marxiste et de l'ethnologie structuraliste. Au lieu de couper l'œuvre de Marx en deux, à l'image des althussériens, Baudrillard lit alors Marx avec les textes de Saussure, de Jacobson et de Barthes en tête. Baudrillard ne peut pas être althussérien, à la différence de Godelier, car il propose sa propre synthèse entre marxisme et structuralisme, qui éviterait justement de faire de la modernité capitaliste une nécessité historique, et donc de faire du mode de vie occidental une finalité objective de l'humanité, quand bien même serait-elle dépassée par un communisme à venir.

Cette problématique fait écho avec une autre note, courte, mais très intéressante sur un penseur très proche de Jean Baudrillard, à savoir le canadien Marshall McLuhan. Parmi la bibliothèque annotée de Baudrillard, il y a des ouvrages, mais il y a aussi bien sûr des revues. On y retrouve certaines des plus importantes revues de l'époque : Les Temps modernes, Critique, Tel Quel, Arguments, L'Homme, Communications, La Nouvelle Revue de Psychanalyse, et bien d'autres. Néanmoins, c'est un tout autre type de revue dans lequel a publié McLuhan (1969), et sur lequel Baudrillard a pris quelques notes, puisqu'il s'agit de la fameuse interview du théoricien des medias pour la revue Playboy intitulé : «a candid conversation with the high priest of popcult and metaphysician of media » ( « une conversation candide avec le grand prêtre du culte pop et le métaphysicien des médias »), et dont Baudrillard a arraché les pages avant 
de les glisser dans l'ouvrage très connu du canadien : Pour comprendre les médias (MCLUHAN, 1968, p. 66). Dans un des passages de l'interview, McLuhan remarque en 1969 le paradoxe d'une "retribalisation " culturelle de la jeunesse blanche nord-américaine (on est alors en plein mouvement hippy), alors qu'au même moment la société nord-américaine en appelle à une uniformisation culturelle des afro-américaines et des amérindiens. Baudrillard ([s.d., s.p.]) écrit en marge : «Valable pour tout le procès de colonisation/civilisation. Aujourd'hui, toutes les structures submergées resurgissent $»$. On comprend ainsi mieux le fort doute que pouvait manifestait Baudrillard dans l'analyse de l'opposition entre modernité capitaliste occidentale et sociétés extra-européennes. Moins qu'une succession évolutionniste de formes de société, où l'on passerait d'un stade tribal à un stade féodal, puis monarchique, puis capitaliste, etc., Baudrillard se rattache à une conception non-linéaire de l'histoire humaine, dans lequel peut surgir de l'inédit. Le système économique capitaliste ne s'est pas substitué aux mondes traditionnels des peuples anciens ou étrangers, il les a seulement submergés. Le système économique mondial n’a pas effacé le monde qui lui a précédé, il s'est juste imposé comme une surface généralisée des échanges mondiaux, système universel de "transparence ", qui certes a le monopole de la circulation des signes et de l'apparence, mais n'est néanmoins qu'une surface, un « écran total » qui, pour être omniprésent, n'en est pas moins incapable d'abolir les altérités qu'il occulte dans cet acte de submersion. Ce faisant, il n'est guère surprenant que cette altérité, que l'on croyait disparue, mais qui était seulement recouverte, revienne à la surface, au moment même où un développement économique sans fin, et donc sans finalité, s'est imposé par la force, dans ce que Jean Baudrillard a appelé : « la violence du mondial » (BAUDRILLARD, 2002, pp. 63-83). Ainsi, les doutes du jeune Baudrillard quant à une linéarité de l'histoire se trouvent confirmés par les événements du début du XXIe siècle que le philosophe commente à la fin de sa vie. Jean Baudrillard est vraiment un auteur qui nous invite à nous mettre à l'écoute de la singularité du temps présent, plutôt que d'assimiler l'inconnu au déjà connu.

L'analyse intégrale des notes de Baudrillard, dont je ne cite ici que quelques exemples, est évidemment impossible dans le temps qui m'est imparti, et implique un travail de recherche sur le long terme. Pour conclure, je terminerai sur deux notes, qui m'ont particulièrement ému. J'ai en effet retrouvé, à la fin du sommaire de la version originale 
de Aurore de Nietzsche, Morgenröthe, cette phrase écrite en allemand par Baudrillard, et que l'on trouve au tout début de son dernier essai, Le Pacte de lucidité, à mon sens son testament philosophique. Sur le livre de Nietzsche, Baudrillard ([s.d., s.p.]) a écrit : « Existenz ist nicht Alles. Sie ist sogar am Allerwenigsten/ am Allergeringsten ». En français dans le texte, et dans Le Pacte de lucidité (BAUDRILLARD, 2004, p. 12), cela donne : «L'existence n'est pas tout - c'est même la moindre des choses ». Il intéressant de voir que, pour écrire cette phrase en allemand, Baudrillard a hésité entre l'adjectif « wenig », qui signifie « peu », et l'adjectif « gering », qui signifie « faible». « L'existence » est certes ce « peu » d'être qui fait que nous sommes, mais elle est aussi quelque chose de « faible », une fragilité consubstantielle sur lequel repose notre être. L'être humain se fonde sur cette fragilité, et exister c'est être cette fragilité, et ne jamais cesser de l'expérimenter.

En écho avec cette idée, je pense à la note de Baudrillard en marge de l'article de Jean-François Lyotard (1971), publié dans le premier numéro de la Revue d'esthétique en 1968, et qui sera compilé dans son grand livre Discours, Figure. Les réflexions annotées par Baudrillard seront d'ailleurs reprises par celui-ci, après réécriture, dans le fameux passage sur les anagrammes de Saussure à la fin de L'échange symbolique et la mort. Baudrillard écrit :

[...] le « vrai » texte poétique est celui dont le texte manifeste et cohérent est pourtant aussi sans reste par rapport à une matrice qui elle par contre n'est pas d'ordre conscient et discursif. La pensée de poésie (Dichtunggedanke) n'est pas un texte lisible, ni quelque chose comme une formule (une clef !) référent positif caché, mais peut-être RIEN, le néant, l'absence à laquelle le texte retourne (BAUDRILLARD, [s.d., s.p.]).

On retrouve ici la passion originelle de Baudrillard pour la poésie. La poésie, selon le philosophe, modifiant la fonction de la langue, n'est pas de l'ordre de l'interprétation, ou de la pensée intellectuelle. Elle est une pure apparition du " néant », de "l'absence », du vide au sein de toute chose, et vide sans lequel il ne peut y avoir ni son, ni musique. Cette pensée du « rien », du vide, nous renvoie bien sûr à la philosophie chinoise, au Tao, mais elle est également au cœur de la pensée du catharisme, qui apparaît au Moyen-Age autour d'une polémique théologique avec l'Eglise catholique sur la définition du néant ${ }^{6}$ dans le «Prologue » de l'Evangile

6 Je renvoie là-dessus à Serge Pey (2009).

Remate de Males, Campinas-SP, v.39, n.2, pp. 867-881, jul./dez. $2019-878$ 
de Jean. Nous savons que Baudrillard s'est constamment revendiqué du manichéisme et del'hérésie cathare, comme il l'affirme dans D’un fragment l'autre (BAUDRILLARD, 2001, p. 86), ainsi que dans une interview pour le journal allemand Der Spiegel en 2002.7 Il fut d'ailleurs un lecteur du grand spécialiste du catharisme : René Nelli ${ }^{8}$. Et si, lorsqu'est évoquée l'hérésie cathare, on pense le plus souvent à l'Occitanie, et à la croisade contre les Albigeois, il n'en demeure pas moins que Reims, la ville natale de Jean Baudrillard, fut également une haute place de l'hérésie. Et il est très probable que Baudrillard le savait, et que ce fut même une des raisons de son attachement pour cette pensée persécutée. Ainsi, avec ce travail de recherche sur la bibliothèque de Jean Baudrillard, j'aurai au moins défendu la bibliothèque d'un hérétique. C'est toujours une de plus qui n’aura pas été brulé par les Inquisitions, celles d'hier comme celles d'aujourd'hui.

\section{$\overline{\text { RÉFÉRENCES }}$}

ALTHUSSER, Louis ; BALIBAR, Etienne ; ESTABLET, Roger ; MACHEREY, Pierre ; RANCIERE, Jacques. Lire le Capital. Tome 1 e 2. Paris : Librairie François Maspero, 1965 .

ARTAUD, Antonin. Le théâtre et son double. Paris : Editions Gallimard, 1964.

BADIOU, Alain. L'Ethique. Paris : Hatier, 1993.

BARTHES, Roland. Mythologies. Paris : Editions, 1957.

BACKLES-CLEMENT, Catherine ; COPANS, Jean ; GODELIER, Maurice ; TORNAY, Serge. L’Anthropologie : Science des sociétés primitives ? Paris : Editions E.P, 1971.

BAUDRILLARD, Jean. Le Miroir de la production ou l'Illusion critique du matérialisme historique. Paris : Casterman, 1973.

BAUDRILLARD, Jean. Le Système des objets. Paris : Editions Gallimard, 1978.

BAUDRILLARD, Jean. Simulacres et simulation. Paris : Editions Galilée, 1981, p.10.

BAUDRILLARD, Jean. La Société de consommation. Paris : Editions Gallimard, 1986.

7 « I love the world of the Cathars because I am Manichaean » (SMITH; CLARCK, 2015, p. 178).

8 J'ai notamment retrouvé dans la bibliothèque, même s'ils ne sont pas annotés : René Nelli (1953), Spiritualité de l'hérésie : Le catharisme, et du même auteur : La Philosophie du catharisme, Le dualisme radical du XIIIe siècle (NELLI, 1975).

Remate de Males, Campinas-SP, v.39, n.2, pp. 867-881, jul./dez. 2019 - 879 
BAUDRILLARD, Jean. L'Échange impossible. Paris : Editions Galilée, 1999.

BAUDRILLARD, Jean. D'un fragment l'autre. [Entretiens avec François L'Yvonnet]. Paris : Editions Albin Michel S.A, 2001, pp. 17 e p. 86.

BAUDRILLARD, Jean. L'Esprit du terrorisme. Paris : Editions Galilée, 2002a.

BAUDRILLARD, Jean. Power Inferno. Paris : Editions Galilée, 2002b, pp.63-83.

BAUDRILLARD, Jean. Le Pacte de lucidité ou l'intelligence du Mal. Paris : Editions Galilée, 2004, p. 12.

BAUDRILLARD, Jean. Le Complot de l'art. Paris : Editions Sens \& Tonka, 2005.

BAUDRILLARD, Jean. Fond privé Marine Baudrillard. Paris : s.d., s.p.

BAUDRILLARD, Jean ; GUILLAUME, Marc. Figures de l'altérité. Paris : Editions Descartes \& Cie., 1998.

BENJAMIN, Walter. Angelus Novus, Ausgewählte Scriften 2. Frankfurt : Suhkamp Verlag, 1966.

BRECHT, Bertolt. Dialogues d'exilés. Paris : L’Arche, 1965.

DELEUZE, Gilles ; GUATTARI, Felix. L’Anti-CEdipe, Capitalisme et schizophrénie. Paris : Minuit, 1972.

GUILLAUME, Valérie. Jean Baudrillard et le Centre Pompidou. Une biographie intellectuelle. Paris/Lormont : Centre Pompidou/Editions Le bord de l'Eau, 2013.

LEVI-STRAUSS, Claude. Anthropologie structurale. Paris : Plon, 1958.

LYOTARD, Jean-François. Discours, Figure. Paris : Klincksieck, 1971.

L'YVONNET, François. Jean Baudrillard. Paris : Editions de l'Herne, 2004, pp. 293-315.

MARX, Karl. Grundrisse der Kritik der politischen Ökonomie. Berlin : Dietz Verlag, 1953.

MARX, Karl. Manuscrits de 1844. Paris : Editions Sociales, 1962.

MARX, Karl. Un chapitre inédit du Capital. Paris : Union générale d'éditions, 1971.

MARX, Karl ; ENGELS, Friedrich. L'Idéologie allemande. Paris : Editions sociales, 1968.

MCLUHAN, Marshall. Pour comprendre les média. Paris : Editions H M H/Ltée, 1968.

MCLUHAN, Marshall. Marshall McLuhan : A Candid Conversation with the High Priest of Popcult and Metaphysician of Media. Playboy [Interview]. Chicago : $\mathrm{HMH}$ Publishing Co., 1969. 
NELLI, René. Spiritualité de l'hérésie : le catharisme. Toulouse/Paris : Privat, PUF, 1953.

NELLI, René. La Philosophie du catharisme. Le Dualisme radical du XIIIe siècle. Paris : Editions Payot, 1975 .

PENOT-LACASSAGNE, Olivier. Back to Baudrillard. Paris : CNRS Editions, 2015, pp. 135-136.

PEY, Serge. Lettres posthumes à Octavio Paz depuis quelques arcanes majeures du tarot. Paris : Jean-Michel Place, 2003.

PEY, Serge. Nihil et Consolamentum. Toulouse : Délit Edition, 2009.

RANCIERE, Jacques. La Haine de la démocratie. Paris : La Fabrique Editions, 2005.

SHALLINS, Marshall. Stone Age Economics. Chicago : Aldine-Atherton, 1972.

SIMONDON, Gilbert. Du Mode d'existence des objets techniques. Paris : Editions Montaigne, 1958.

SMITH, Richard G. ; CLARCK, David B. Jean Baudrillard : From Hyperreality to Disappearance : Uncollected Interviews. Edinburgh : Edinburgh University Press, 2015 , p.178.

SONTAG, Susan. La Photographie. Paris : Editions du Seuil, 1979.

SLOTERDIJK, Peter. Im selben Boot, Versuch über die Hyperpolitik. Berlin : Suhrkamp Verlag, 1995 .

ZIZEK, Slavoj. Welcome to the Desert of the Real. London, NY : Verso, 2002.

Recebido: 25/05/2019

Aceito: 12/07/2019

Publicado: 27/11/2019

Remate de Males, Campinas-SP, v.39, n.2, pp. 867-881, jul./dez. 2019 - 881 